treatment before quitting might be important. In fact, clinical data show that treatment of patients with a nicotine patch for 2 weeks before quitting is associated with a statistically significant increase in abstinence of $79 \%$ over postcessation use only. ${ }^{9}$ The effectiveness of pre-cessation use is best explained by reductions in reward from smoking weakening conditioned responses, but mediation analysis of the effect of pre-cessation NRT or varenicline on cigarette reward would be needed to confirm this. Whatever the mechanism, pre-cessation use of nicotine patch should be considered in some smokers, although further large trials are probably necessary before precessation use of NRT becomes the norm.

The results of the study by Aubin et al are to be welcomed because varenicline provides another option for smokers wanting to quit. We can now provide clearer statements to our patients that varenicline is the best single pharmacotherapy to assist an attempt to quit. Although varenicline is more effective than the current standard patch, there are good reasons to assume that NRT taken before quit day and in combination might be of similar efficacy. NRT may become even more effective if newer, higher dose, rapid release formulations become available. In my view, however, in varenicline we are close to an optimal treatment for nicotine dependence, but stopping smoking involves more than treating nicotine dependence. Most people who successfully reach the end of treatment will relapse to smoking. While taking longer courses of varenicline may prevent some relapse, lifetime varenicline is unlikely to be a major solution. Instead, we need to develop cognitive-behavioural interventions together with judicious use of various medications. Until then, varenicline is a welcome treatment option that is likely to prove popular with clinicians and patients.

Funding: PA is funded by the National Institute for Health Research (NIHR) but the views expressed are not necessarily those of the NIHR or the NHS.

Competing interests: PA has done consultancy work for the pharmaceutical and biotechnology industries that led to payments to him and his institution. This includes work for companies providing smoking cessation medication including McNeil, who manufacture NRT, and Pfizer, the makers of varenicline.

Thorax 2008:63:666-668. doi:10.1136/thx.2008.096081

\section{REFERENCES}

1. Aubin J-H, Bobak A, Britton JR, et al. Varenicline versus transdermal nicotine patch for smoking cessation: results from a randomised, open-label trial. Thorax 2008;63:717-24.

2. Jorenby DE, Hays JT, Rigotti NA, et al. Efficacy of varenicline, an alpha4beta2 nicotinic acetylcholine receptor partial agonist, vs placebo or sustained-release bupropion for smoking cessation: a randomized controlled trial. JAMA 2006;296:56-63.

3. Gonzales D, Rennard SI, Nides M, et al. Varenicline, an alpha4beta2 nicotinic acetylcholine receptor partia agonist, vs sustained-release bupropion and placebo for smoking cessation: a randomized controlled trial. JAMA 2006;296:47-55

4. Stapleton JA, Watson L, Spirling LI, et al. Varenicline in the routine treatment of tobacco dependence: a pre-post comparison with nicotine replacement therapy and an evaluation in those with mental illness. Addiction 2008;103:146-54

5. National Institute for Health and Clinical Excellence (NICE). Final appraisal determination for varenicline for smoking cessation. 2007. http:// guidance.nice.org.uk/page.aspx?0 $=431473$ (accessed 3 June 2008)

6. Hughes JR, Stead LF, Lancaster T. Antidepressants for smoking cessation. Cochrane Database Syst Rev 2007; (1):CD000031.

7. National Institute for Health and Clinical Excellence (NICE). Guidance on smoking cessation. London: NICE, 2008.

8. Cahill K, Stead LF, Lancaster T. Nicotine receptor partial agonists for smoking cessation. Cochrane Database Syst Rev 2007;(1):CD006103

9. Stead LF, Perera R, Bullen C, et al. Nicotine replacement therapy for smoking cessation. Cochrane Database Syst Rev 2008;(1):CD000146.

10. Stapleton JA. Is varenicline cost-effective enough to be funded by the NHS now? 2006. http://www.scsrn. org/policy_guidance/Narenicline_NHS_CE.pdf (accessed 16 Feb 2008).

11. Etter JF, Stapleton JA. Nicotine replacement therapy for long-term smoking cessation: a meta-analysis. Tob Control 2006;15:280-5.

12. National Institute for Health and Clinical Excellence. Guidance on the use of nicotine replacement therapy (NRT) and bupropion for smoking cessation. 2002. http://www.nice.org.uk/nicemedia/ pdf/NiceNRT39GUIDANCE.pdf laccessed 16 Feb 2008).

13. West R, DiMarino ME, Gitchell J, et al. Impact of UK policy initiatives on use of medicines to aid smoking cessation. Tob Control 2005;14:166-71.

14. Shiffman S, West RJ, Gilbert DG. Recommendation for the assessment of tobacco craving and withdrawal in smoking cessation trials. Tob Res 2004;6:599-614.

15. Piasecki TM. Relapse to smoking. Clin Psychol Rev 2006;296:196-215.

16. Johnstone E, Brown K, Saunders C, et al. Level of nicotine replacement during a quit-smoking attempt. Tob Res 2004:6:377-9.

17. Stapleton JA, Russell MA, Feyerabend C, et al. Dose effects and predictors of outcome in a randomized trial of transdermal nicotine patches in general practice. Addiction 1995;90:31-42.

18. Shiffman S, Ferguson SG, Gwaltney CJ. Immediate hedonic response to smoking lapses: relationship to smoking relapse, and effects of nicotine replacement therapy. Psychopharmacology (Berl) 2006; 184:608-18.

\title{
Lung transplant and cystic fibrosis: what's new from the UK and France?
}

\section{Peadar G Noone}

Although the median survival for patients with cystic fibrosis (CF) has improved steadily over the past several decades, many patients go on to develop respiratory failure from progressive lung disease, eventually requiring lung transplantation for extended survival. ${ }^{1}$ Although many years have elapsed since the first lung transplants were performed for CF, the

Correspondence to: Dr Peadar G Noone, Pulmonary Division, CB\# 7020, UNC Chapel Hill, NC 27599-7020, USA; pnoone@med.unc.edu field is not without controversy. ${ }^{2}$ The paper recently published by Liou et al is one recent example, suggesting that lung transplant for most children with CF under 18 years of age offers no survival advantage. ${ }^{2}$ The complex statistical methodology and conclusions have since been challenged and rebutted by several lung transplant experts. ${ }^{3-5}$ Controversial issues like this often reflect the shortage of randomised controlled trials for many aspects of lung transplant. Although there is much published material related to lung transplant, many protocols are based on retrospective data, or are rather centre or region specific. Although impure because of multiple confounding factors, such as small sample sizes, and varying surgical and medical protocols, these data do provide a reasonable template to formulate and update lung transplant protocols.

Two such datasets ${ }^{67}$ are published in this issue of Thorax (see pages 725 and 732). The first represents the experience of a single centre in the UK, accounting for a large proportion of lung transplants for CF in Britain, ${ }^{6}$ while the second paper from France addresses a difficult issuethat is, the risks of performing lung transplantation in patients infected with Burkholderia cepacia complex (BCC) organisms. The papers are complementary: the UK experience is a general report, excluding a detailed analysis of outcomes in patients with BCC (these 
data are promised shortly), while the French paper is BCC specific.

The Newcastle Lung Transplant Centre can take pride in its achievements as a transplant programme for patients with $\mathrm{CF}$, having been at the forefront of the field for almost 20 years. The data in this issue of Thorax are largely descriptive, but likely represent the largest single centre report for CF. ${ }^{6}$ A total of 176 patients with CF have been transplanted since 1989, representing $\sim 30 \%$ of the total transplanted cohort $(n=576)$ in Newcastle. Few were paediatric patients $(n=9)$ with none less than age 12 years. ${ }^{2}$ There were a few heart-lung transplants $(\mathrm{n}=4)$, one living related lung transplant, one retransplant (a difficult issue) and, interestingly for CF, two single lung transplants for unusual clinical problems. A pure transplant specialist might want more specifics on the medical and surgical aspects of the procedure and follow-up, but some interesting approaches are worthy of comment. Cardiopulmonary bypass was used in all cases, with an emphasis on the technique of "simultaneous reperfusion of both lungs" to reduce circulatory stress on an individual lung (no data are presented to show any benefit on outcomes). Importantly, prior pneumothorax $(n=21)$, some with prior medical or surgical pleurodesis, was not regarded as a contraindication, nor did it influence outcomes. Non-invasive ventilation pretransplant $(\mathrm{n}=22)$ did not adversely influence outcome, and apparently no patient with CF was intubated pretransplant (other than the "re-do" patient). Overall survival for the cohort compares very well with other published data, as well as Newcastle's own non-CF outcomes: $82 \%$ survival at 1 year and $62 \%$ at 5 years; $84 \%$ at 1 year if one excludes the $B$ cenocepacia patients. Most deaths were from infection (a large number in the $B$ cenocepacia group, especially early deaths; see Boussaud and colleagues ${ }^{7}$ in this issue), and obliterative bronchiolitis (BOS), all consistent with other published series. As previously published by other groups, Pseudomonas resistance patterns did not adversely influence outcome. Although there is apparently no policy to exclude patients with non-tuberculous mycobacteria, none was transplanted during the review period, which is curious, given the published prevalence of such organisms in CF in the US and Europe. ${ }^{89}$ In the discussion, the authors stress their operative techniques of reanastomosing the bronchi, which they believe accounts for a lower than expected complication rate of $2 \%$. There was a
$73.5 \%$ freedom from BOS at 5 years. Although not specifically mentioned, this might relate to the use of an immunosuppressive induction protocol (anti-thymocyte globulin). This may be important, since ISHLT data do not show any reduction in $\mathrm{BOS}$ rates with use of induction protocols, although some reports show a reduction in acute rejection rates. ${ }^{10}$ Reported long term morbidities are not surprising, with renal impairment common, but the centre employs aggressive "renal preserving measures" such as reducing ciclosporin or tacrolimus doses at 1 year and avoidance of systemic aminoglycosides after transplant, not an easy feat given the plethora of resistant CF organisms prevalent in this population. No outcome data are presented to support this approach, although it seems reasonable.

Lung transplant for patients with CF infected with BCC organisms has long been controversial. Many transplant programmes, particularly in the USA, have stopped offering lung transplant to these patients based on a higher than expected risk of death postoperatively. In other parts of the world, there is no similar absolute moratorium. Some programmes distinguish between patients with BCC in general, and those with $B$ cenocepacia strains, excluding only the latter, as they have the highest risk of performing poorly post-transplant compared with non-cenocepacia BCC. ${ }^{11-14}$ Other programmes take referrals on a case by case basis, especially those with affiliated CF centres and large numbers of patients with $B$ cenocepacia, presumably reflecting the notion that these patients also deserve the opportunity of extended survival via lung transplantation, despite the published risks. That seems to be the case in relation to the second transplant related paper in this issue of Thorax, where the post-transplantation outcomes of CF patients with BCC infections in two French centres accepting patients infected with these bacteria are reported. ' Twenty-two patients with BCC (of a total of 247 patients with CF) were transplanted. Eight had $B$ cenocepacia (previously known as genomovar III) whereas most of the remainder were infected with $B$ multivorans $(\mathrm{n}=11)$. As the numbers are small, it is hard to make definitive conclusions, but the primary result that emerges is that, as reported previously, patients with BCC have outcomes comparable with other CF patients, but the small subgroup of patients with $B$ cenocepacia has poorer survival. There were several early deaths in the cenocepacia group (table 1), with few long term survivors.

So why is the paper of interest, if it is more of the same, one might ask? One suspects the authors are publishing the data at least in part to be provocative and to encourage transplant programmes to keep an open mind about transplanting patients with $B$ cenocepacia. The issue is almost philosophical, given the consistency of the data over the years. It is clear from the paper that the authors are among those devoted to the notion of keeping the option of lung transplant for patients with these risk laden organisms, which is laudable, given that these patients face death if denied transplant in the setting of very severe lung disease. Most centres, as in this report, have a few anecdotal experiences of patients with $B$ cenocepacia having long term survival post-transplant, and this experience often drives the local process (similarly, even a small number of very poor outcomes can have the opposite effect). Where the prevalence of $B$ cenocepacia is high, as for example in Toronto, the transplant team there has adopted an aggressive approach of prolonged post-transplant antibiotic therapy in an effort to improve outcomes, a strategy employed by the authors of the current paper. ${ }^{13}$ At the University of North Carolina, after adopting a Toronto-like prolonged antibiotic protocol, such patients continued to be offered lung transplant (up to 2006 ), until the postoperative survival data became so inferior to non- $B$ cenocepacia infected patients as to make it difficult to continue. ${ }^{15}$ Over the past few years, most centres in the US appear to have discontinued taking referrals along similar lines.

Personal communications from Australia suggest that although there is no absolute moratorium on lung transplant for patients with $B$ cenocepacia, most centres scrutinise referred patients very carefully for any added risk factors or comorbidities. As evidenced by the Newcastle and French data ${ }^{67}$ in this issue of Thorax, some European centres continue to offer lung transplant for patients with CF and $B$ cenocepacia, even with the reduced survival as presented. As anyone involved in lung transplant and CF knows, it can be difficult for transplant teams to deny apparently good (otherwise) candidates. The French authors emphasise the need to examine measures other than survival (the ultimate transplant outcome); for example, quality of life, and offer a recent increase in organ donation in France (improving the donor/ wait list ratio), with shortened wait 
times, as one plausible reason why all patients, including high risk ones, need to be given the opportunity for transplant. On the other hand, such a sympathetic approach is not always possible, especially in systems where third party payers, and other outside agencies, regulate the activity of transplant centres through accreditation procedures, and where survival is the ultimate measure of a centre's performance, with no credit allowed for taking on high risk patients.

No doubt, many in the field of lung transplant will continue to argue back and forth about these issues. At least one thing is again clear from the data; as with previously published series, where appropriate, non- $B$ cenocepacia BCC patients with CF should not be excluded from the opportunity to be evaluated for lung transplant.

Acknowledgements: The author gratefully acknowledges the helpful comments of Dr Scott Donaldson during the writing of this manuscript.

Competing interests: None.

Thorax 2008;63:668-670. doi:10.1136/thx.2008.099622

\section{REFERENCES}

1. CFF. 2007. Cystic Fibrosis Foundation Patient Registry. 2006 Annual Report to the Center Directors. Bethesda: CFF, 2006.

2. Liou TG, Adler FR, Cox DR, et al. Lung transplantation and survival in children with cystic fibrosis. N Engl J Med 2007;357:2143-52.

3. Sweet SC, Aurora P, Benden C, et al. Lung transplantation and survival in children with cystic fibrosis: solid statistics_-flawed interpretation. Pediatr Transplant 2008; 12:129-36.

4. Egan TM. Solid benefit of lung transplantation for some children with cystic fibrosis. Pediatr Transplant 2008:12:125-8.

5. Aurora P, Spencer H, Moreno-Galdo A. Lung transplantation in children with cystic fibrosis. A view from Europe. Am J Respir Crit Care Med 2008;177:935-6.

6. Meachery G, De Soyza A, Nicholson A, et al. Outcomes of lung transplantation for cystic fibrosis in a large UK cohort. Thorax 2008:63. 725-31.

7. Boussaud V, Guillemain R, Grenet D, et al. Clinical outcome following lung transplantation in patients with cystic fibrosis colonised with Burkholderia cepacia complex: results from two French centres. Thorax 2008;63:732-7.

8. Olivier KN, Weber DJ, Wallace RJ Jr, et al. Nontuberculous mycobacteria: I. Multicenter prevalence study in cystic fibrosis. Am J Respir Crit Care Med 2003:167:828-34.
9. Jordan PW, Stanley T, Donnelly FM, et al Atypical mycobacterial infection in patients with cystic fibrosis: update on clinical microbiology methods. Lett Appl Microbiol 2007:44: 459-66.

10. Ailawadi G, Smith PW, Oka T, et al. Effects of induction immunosuppression regimen on acute rejection, bronchiolitis obliterans, and survival after lung transplantation. J Thorac Cardiovasc Surg 2008;135:594-602.

11. De Soyza A, McDowell A, Archer L, et al. Burkholderia cepacia complex genomovars and pulmonary transplantation outcomes in patients with cystic fibrosis. Lancet 2001: 358:1780-1.

12. Aris RM, Routh JC, Lipuma JJ, et al. Lung transplantation for cystic fibrosis patients with Burkholderia cepacia complex. Survival linked to genomovar type. Am J Respir Crit Care Med 2001:164:2102-6.

13. Chaparro C, Maurer J, Gutierrez C, et al. Infection with Burkholderia cepacia in cystic fibrosis: outcome following lung transplantation. Am J Respir Crit Care Med 2001;163:43-8.

14. Alexander BD, Petzold EW, Reller LB, et al. Survival after lung transplantation of cystic fibrosis patients infected with Burkholderia cepacia complex. Am J Transplant 2008;8 1025-30.

15. Egan TM, Detterbeck FC, Mill M, et al. Impact of Burkholderia species on outcome of lung transplantation for CF patients. Pediatr Pulmonol 2005:28(Suppl):365

\section{Gene modulation and severity of lung disease in cystic fibrosis}

Progressive lung disease is an important cause of mortality and morbidity in patients with cystic fibrosis (CF). The severity and progression of lung disease, which has a poor correlation with CF transmembrane conductance regulator (CFTR) genotype, is thought to be modulated by secondary genetic factors known as CF modifiers. Mannose binding lectin 2 (MBL2) and transforming growth factor $\beta 1$ (TGF $\beta 1$ ) genes are among the pulmonary modifiers associated with an earlier onset of lung infection and a more rapid decline in lung function. The objectives of this study were to evaluate the role of MBL2 and TGF $\beta 1$ as pulmonary modifiers and to analyse interactions between them.

A cohort of 1019 patients with CF, representative of the general CF population in Canada, were recruited from specialised CF clinics from 2003 to 2006. At every clinic visit sputum cultures and lung function tests were performed and the age at the time of the first positive respiratory culture for Pseudomonas aeruginosa was recorded. Plasma and DNA samples were obtained for measurement of MBL2 protein levels and for MBL2 and TGF $\beta 1$ genotyping.

The study showed that children with CF with MBL2 deficiency acquired Pseudomonas infection earlier and also had a faster rate of decline in lung function than those with intermediate or high levels of MBL2. Those who had high expressing variant of TGF $\beta 1$ genotype had more severe lung disease, suggesting a complex interaction between these two genes.

Further studies are needed to evaluate the effects of other pulmonary modifiers and their interactions in what is an increasingly complex genetic disease. It is also not known how these modifiers affect other aspects of CF. Gaining insight into the mechanism of interaction between these and other pulmonary modifiers will help in developing modifier-based therapies.

Dorfman R, Sandford A, Taylor C, et al. Complex two-gene modulation of lung disease severity in children with cystic fibrosis. J Clin Invest 2008;118:1040-9

\section{A Pillai}

Correspondence to: A Pillai, Specialty Trainee, Gloucestershire Royal Hospital, Gloucester, UK; anilkumarpillai@doctors.org.uk 